\title{
All exceptional surgeries on alternating knots are integral surgeries
}

\author{
KAZUHIRO ICHIHARA
}

\begin{abstract}
We show that all non-trivial exceptional surgeries on hyperbolic alternating knots in the 3-sphere are integral surgeries.
\end{abstract}

$57 \mathrm{M} 50$

\section{Introduction}

By a Dehn surgery on a knot (ie, an embedded circle in a 3-manifold), we mean the following operation to create a new 3-manifold from a given one and a given knot; remove an open tubular neighborhood of the knot, and glue a solid torus back. Here and hereafter in this paper, all 3-manifolds are assumed to be orientable.

The well-known Hyperbolic Dehn Surgery Theorem due to Thurston [31, Theorem 5.8.2] says that, each hyperbolic knot (ie, a knot with hyperbolic complement) admits only finitely many Dehn surgeries yielding non-hyperbolic manifolds. In view of this, such finitely many exceptions are called exceptional surgeries.

In this paper, we consider exceptional surgery on hyperbolic alternating knots in the 3sphere $S^{3}$. A knot in $S^{3}$ is called alternating if it admits a diagram with alternatively arranged over-crossings and under-crossings running along it. Note that Menasco showed in [22] that an alternating knot in $S^{3}$ is hyperbolic unless it is a $(2, p)$-torus knot; that is, the knot isotoped to the $(2, p)$-curve on the standard embedded torus in $S^{3}$.

Here let us recall fundamental terminologies about Dehn surgery. See Rolfsen [28] in details for example. As usual, by a slope, we mean the isotopy class of a non-trivial unoriented simple closed curve on a torus. Consider the slope on the peripheral torus of a knot $K$ which is represented by the curve identified with the meridian of the attached solid torus via the surgery. Then we can see that Dehn surgery on $K$ is characterized by the slope, which we call the surgery slope. When $K$ is a knot in $S^{3}$, by using the standard meridian-longitude system, slopes on the peripheral torus are parametrized by rational numbers with $1 / 0$. For example, the meridian of $K$ corresponds to $1 / 0$ and 
the longitude to 0 . By the trivial Dehn surgery on $K$ in $S^{3}$, we mean the Dehn surgery on $K$ along the meridional slope $1 / 0$. Thus it yields $S^{3}$ again, which is obviously exceptional, when $K$ is hyperbolic. We say that a Dehn surgery on $K$ in $S^{3}$ is integral if it is along an integral slope. This means that the curve representing the surgery slope runs longitudinally once.

Then our main theorem is as follows.

Theorem 1.1 On a hyperbolic alternating knot in the 3-sphere, all non-trivial exceptional surgeries are integral.

In general, it is conjectured that, on a hyperbolic knot in $S^{3}$, all non-trivial exceptional surgeries are integral or half-integral. See Kirby [18, Problem 1.77, (A) Conjecture (3)]. Only known example of hyperbolic knots in $S^{3}$ admitting non-integral exceptional surgeries are the knots given by Eudave-Muñoz in [9]. Also see Section 3.2. Thus Theorem 1.1 implies that the knots given by Eudave-Muñoz in [9] are all non-alternating.

Also, as an immediate corollary, we have the following.

Corollary 1.2 A hyperbolic alternating knot in the 3-sphere admits at most 10 exceptional surgeries.

Proof Let $K$ be a hyperbolic alternating knot in the 3-sphere. Then, by Theorem 1.1, all non-trivial exceptional surgeries are integral. On the other hand, as an immediate corollary to Ichihara [16, Theorem 1.1], it is shown that; on any hyperbolic knot in $S^{3}$, there are at most 9 integral exceptional surgeries. Thus, together with the trivial surgery, $K$ admits at most 10 exceptional surgeries.

This concerns the famous Gordon conjecture; there exist at most 10 exceptional surgeries on each hyperbolic knot. See [18, Problem 1.77]. Previously, the sharpest known bound was 12, which is obtained as a corollary of the so-called "6-theorem" given by Agol [1, Theorem 8.1] and Lackenby [19] independently. Recently, in [2], Agol announced that there are at most finitely many hyperbolic knots which admit more than eight exceptional surgeries. And, very recently, in [20], Lackenby and Meyerhoff announced the affirmative answer to this conjecture by a combination of new geometric techniques and a rigorous computer-assisted calculation. It should be mentioned that Corollary 1.2 is worth little after Lackenby and Meyerhoff [20], but they do not show the integrality of non-trivial exceptional surgeries. 
Acknowledgments The author was partially supported by Grant-in-Aid for Young Scientists (B), No. 18740038, Ministry of Education, Culture, Sports, Science and Technology, Japan. He would like to thank Shigeru Mizushima and Tetsuya Abe for their comments on the arguments about Lemma 2.4. He also thanks the referee for his/her careful reading.

\section{Proof of Theorem 1.1}

We start with recalling a classification of exceptional surgeries. As a consequence of the famous Geometrization Conjecture, raised by Thurston [32, Section 6, Question 1], and established by recent Perelman's works [25; 26; 27], all closed orientable 3-manifolds are classified as; reducible (ie, containing 2-spheres not bounding 3-balls), toroidal (ie, containing incompressible tori), Seifert fibered (ie, foliated by circles), or hyperbolic (ie, admitting a complete Riemannian metric with constant sectional curvature -1 ). See Scott [29] for a survey. Thus exceptional surgeries are also divided into three types; reducible (ie, yielding a reducible manifold), toroidal (ie, yielding a toroidal manifold), or Seifert fibered (ie, yielding a Seifert fibered manifold).

We first show the following lemma.

Lemma 2.1 If a hyperbolic alternating knot $K$ has a connected prime alternating diagram $D$ satisfying $t(D)>4$, then all non-trivial exceptional surgeries are integral.

The proof of this lemma heavily depends upon the result obtained by Lackenby in [19]. Thus we prepare some terminologies defined and used there. Actually the following are simplified versions of the original definitions. See [19] for full details. Let $D$ be a connected alternating diagram of a knot in $S^{3}$, which we view as a 4-regular graph embedded in $S^{2}$, equipped with "under-over" crossing information. Then $D$ is called prime if each simple closed curve in $S^{2}$ intersecting $D$ transversely in two points divides $S^{2}$ into two discs, one of which contains no crossings of $D$. The twist number of the diagram $D$, denoted by $t(D)$, is defined as the number of twists, which are either; maximal connected collections of bigon regions in $D$ arranged in a row or isolated crossings adjacent to no bigon regions.

Proof of Lemma 2.1 Suppose that a hyperbolic alternating knot $K$ has a connected prime alternating diagram $D$. Then the exterior of $K$ can be given the canonical angled spine arising from $D$. See [19, Section 4] for the definition of the angled spine, and see [19, Section 5] for the construction of the canonical angled spine arising from $D$. From such an angled spine structure, the combinatorial length of a slope on 
(the peripheral torus of) $K$ can be defined. See [19, Section 4] for its definition. Then it follows from [19, Theorem 4.9] that the combinatorial length of the slope $p / q$ on $K$ is at least $|q| \cdot t(D) \cdot \pi / 4$.

On the other hand, also in [19], Lackenby established a combinatorial analogue of the so-called " $2 \pi$-theorem" as [19, Theorem 5.4]. The following is a simplified version of his theorem, together with the affirmative answer to the Geometrization conjecture: Let $K$ be a hyperbolic knot in a closed orientable 3-manifold, and $r$ a slope with combinatorial length more than $2 \pi$ with respect to some angled spine in the exterior of $K$. Then Dehn surgery on $K$ along $r$ must yield a closed hyperbolic 3-manifold.

Therefore, on a hyperbolic alternating knot $K$ with a connected prime alternating diagram $D$ satisfying $t(D)>4$, Dehn surgery along a non-integral slope (ie, slope $p / q$ with $|q| \geq 2$ ) must yield a closed hyperbolic 3-manifold, that is, such a surgery cannot be exceptional.

In the case where $t(D) \leq 4$, we have the following lemma.

Lemma 2.2 If a hyperbolic alternating knot $K$ in $S^{3}$ has a connected prime alternating diagram $D$ satisfying $t(D) \leq 4$, then $K$ must be an arborescent knot.

We can actually determine all the possible prime alternating diagrams.

Here we recall definitions of an arborescent knot and its type. See Wu [33] for full details. By a tangle, we mean a pair with a 3-ball and properly embedded arcs. From two arcs of rational slope drawn on the boundary of a pillowcase-shaped 3-ball, one can obtain a tangle, which is called a rational tangle. A tangle obtained by putting rational tangles together in a horizontal way is called a Montesinos tangle. An arborescent tangle is then defined as a tangle that can be obtained by summing several Montesinos tangles together in an arbitrary order.

Suppose that a knot $K$ in $S^{3}$ is obtained by closing a tangle $T$. If $T$ is a Montesinos tangle, then we call $K$ a Montesinos knot, and if $T$ is an arborescent tangle, then we call $K$ an arborescent knot. For a Montesinos knot, the number of rational tangles forming the corresponding Montesinos tangle is called the length of the Montesinos knot. We denote by $M\left(r_{1}, r_{2}, \ldots, r_{n}\right)$ a Montesinos knot constructed from rational tangles corresponding to rational numbers $r_{1}, r_{2}, \ldots, r_{n}$. In particular, $M\left(1 / q_{1}, 1 / q_{2}, \ldots, 1 / q_{n}\right)$ with integers $q_{1}, q_{2}, \ldots, q_{n}$ is called a pretzel knot of $n$-strands.

In [33], Wu divides all arborescent knots into three types: By the type I knots, we mean two-bridge knots or Montesinos knots of length 3. A bridge index of a knot in $S^{3}$ is defined as the minimal number of local maxima (or local minima) up to ambient 
isotopy. Thus a knot with bridge index 2 is called a two-bridge knot. As remarked before, Menasco showed in [22] that a two-bridge knot is hyperbolic unless it is a $(2, p)$-torus knot. A knot of type II is defined as the union of two Montesinos tangles, each of which is formed by two rational tangles corresponding to $1 / 2$ and a non-integer. All the other arborescent knots are called of type III.

Proof of Lemma 2.2 Suppose that a hyperbolic alternating knot $K$ in $S^{3}$ has a connected prime alternating diagram $D$ satisfying $t(D) \leq 4$. By regarding twists in $D$ (ie, maximal connected collections of bigon regions in $D$ or isolated crossings adjacent to no bigon regions) as fat vertices, we have a planar embedding of a 4-regular graph with at most four vertices. Note that when $t(D)>1$, such a graph has no loops otherwise the graph $D$ would be non-prime.

Then we can tabulate all planar embeddings of such graphs as illustrated in Figure 1 (the case of $t(D) \leq 3$ ) and Figure 2 (the case of $t(D)=4$ ). This is done by using elementary diagrammatic arguments, and so, we omit the details. In the figures, we denote the vertices by small white boxes and the edges by thick lines.
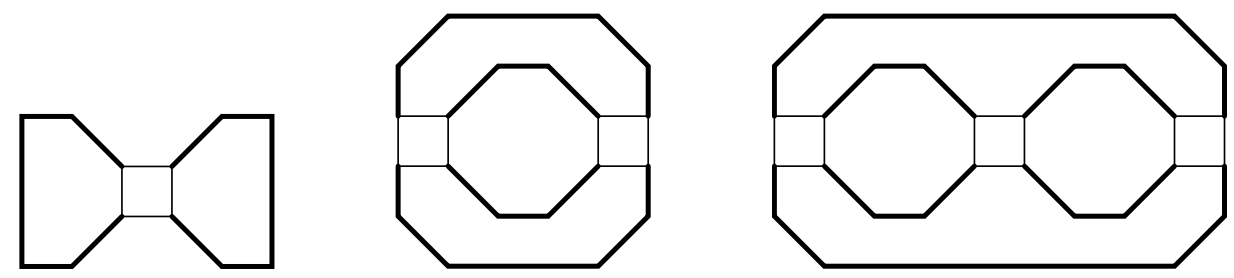

Figure 1

By substituting suitable vertical or horizontal sequences of crossings into the white boxes, we can reconstruct the diagram $D$. Thus it suffices to show that all the prime connected diagrams obtained from the graphs in Figure 1 and Figure 2 represent arborescent knots.

We first consider the graphs in Figure 1. Those three graphs are corresponding to the cases $t(D)=1,2,3$, respectively. In the case where $t(D)=1$ (left in Figure 1), it is clear that the resulting diagrams represent the unknot or $(2, p)$-torus knots, which are two-bridge knots. In the case where $t(D)=2$ (center in Figure 1), by substituting both vertical or both horizontal sequences of crossings into the two white boxes, we have the diagrams representing $(2, p)$-torus knots. On the other hand, if we substitute one vertical and one horizontal sequences of crossings into the two white boxes, we have the diagrams representing two-bridge knots. In the case where $t(D)=3$ (right in Figure 1), if we substitute three vertical sequences of crossings into all the three white 


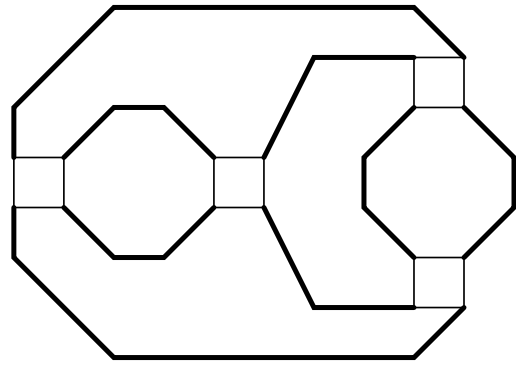

(a)

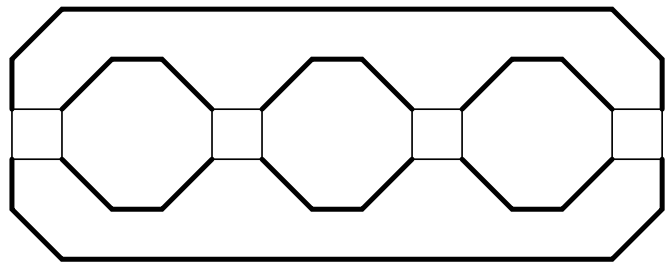

(b)

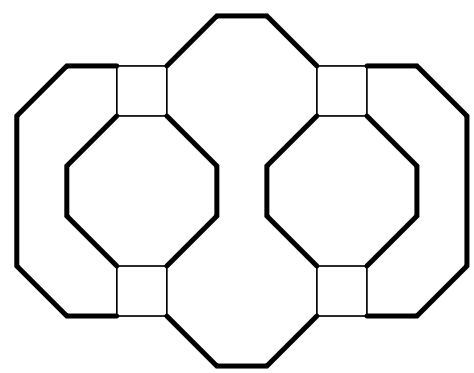

(c)

Figure 2

boxes, then it represents a 3-strand pretzel knot. Otherwise, we see that the diagrams so obtained all represent two-bridge knots (possibly $(2, p)$-torus knots).

We next consider the graphs in Figure 2. Those three graphs are corresponding to the case $t(D)=4$. Note that, from the graph (c), we only have non-prime diagrams by substituting sequences of crossings. Thus we can ignore it.

In the graph (a), there are four boxes; left two and right two. If we substitute both horizontal sequences of crossings into the left two boxes, or both vertical sequences of crossings into the right two boxes, then the diagrams so obtained have the twist number at most three, and so we can ignore these cases. If we substitute vertical and horizontal sequences of crossings into the left two and the right two white boxes simultaneously, we have the diagrams representing the unions of two rational tangles. Such diagrams actually represent two-bridge knots. Now suppose that we substitute two vertical sequences of crossings into the left two white boxes. If we further substitute vertical and horizontal sequences of crossings into the right two boxes, then we obtain a diagram of a Montesinos knot of length three. After taking a mirror image if necessary, it is denoted by $M\left(1 / q_{1}, 1 / q_{2}, 1 /\left(q_{3}+1 / q_{4}\right)\right)$ with $q_{i}>1$ for $1 \leq i \leq 4$. Finally, if we further substitute two horizontal sequences of crossings into the right two white 
boxes, then we obtain a diagram of an arborescent knot of type II or III. In fact, if each one of the two sequences of crossings in the left and right white boxes is a full twist (ie, a pair of crossings), then the knot so obtained is of type II. Otherwise all the knots so obtained are of type III.

In the graph (b), there are four boxes arranged in line. If we substitute two horizontal sequences of crossings into the adjacent two boxes, then the diagrams so obtained have the twist number at most three, and so we can ignore these cases. If we substitute vertical and horizontal sequences of crossings alternatively into the four white boxes, then we have the diagrams representing the unions of two rational tangles. Such diagrams actually represent two-bridge knots. If we substitute four vertical sequences of crossings into the boxes, then we obtain a diagram representing a 4-strand pretzel knot. Finally, it we substitute one horizontal sequences of crossings and three vertical sequences of crossings into the four boxes, then we have a diagram of a Montesinos knot of length three. Actually, after taking a mirror image, we have a Montesinos knot $M(1 / p, 1 / q, n+1 / r)$ with positive integers $p, q, r, n$.

Consequently we have seen that all the prime connected diagrams obtained from the graphs in Figure 1 and Figure 2 represent arborescent knots. This completes the proof of Lemma 2.2.

Thus we can divide the remaining arguments into three cases as follows.

Lemma 2.3 On a hyperbolic two-bridge knot in $S^{3}$, all non-trivial exceptional surgeries are integral.

Proof In fact, in [5], Brittenham and Wu gave a complete classification of the exceptional surgeries on hyperbolic two-bridge knots. From this, we can verify that; on a hyperbolic two-bridge knot, all non-trivial exceptional surgeries are integral. In fact, they showed that among two-bridge knots, only the twist knots (ie, the knots with the corresponding partial fraction decomposition of length two) can admit non-trivial exceptional surgeries, which are actually all integral.

Lemma 2.4 On a hyperbolic alternating Montesinos knot of length 3 in $S^{3}$, all non-trivial exceptional surgeries are integral.

The key ingredient in the proof of this lemma is using essential laminations in 3manifolds, defined by Gabai and Oertel in [11] as follows: We say a lamination $\lambda$ (ie, a co-dimension one foliation of a closed subset of the ambient manifold) is an essential lamination in a 3-manifold $M$ if it satisfies the following conditions. 
(1) The inclusion of leaves of $\lambda$ into $M$ induces an injection between their fundamental groups.

(2) The complement of $\lambda$ is irreducible.

(3) The lamination $\lambda$ has no sphere leaves.

(4) The lamination $\lambda$ is end-incompressible.

More about essential laminations, see Gabai [10] for example.

Proof of Lemma 2.4 In an unpublished preprint [7], Delman gave a construction of essential lamination in a Montesinos knot exterior. See Delman-Roberts [8] for a part of his construction. Actually if the Montesinos knot we consider has the form $M\left(1 / p, r_{1}, r_{2}\right)$ with $p$ and all the denominators of the $r_{i}$ are odd, then the construction given in [8] can be applied in our case. Also see Delman [6] for prototype of the construction in [7]. Further detailed explanations of his construction for alternating Montesinos knots will be appeared in Ichihara-Jong-Mizushima [17].

In particular, Delman showed in [7] that all the Montesinos knots, not of the form $M(x, 1 / p, 1 / q)$ where $x \in\{-1 / 2 n,-1 \pm 1 / 2 n,-2+1 / 2 n\}$ and $p, q$, and $n$ are positive integers, admit essential laminations in their exteriors, which survive after all non-trivial Dehn surgeries. By the claim given in Thistlethwaite [30, Section 4, 2nd paragraph], we see that these exceptions are all non-alternating knots. Precisely he claimed that a Montesinos knot is alternating if and only if its reduced Montesinos diagram, introduced in [21], is alternating. And actually the reduced Montesinos diagrams of the Montesinos knots above are all non-alternating. See [30] and [21] for detail.

Thus, all the alternating Montesinos knots have such essential laminations in the exteriors. By examining his construction, we can verify that each essential lamination so constructed admits at least two disjoint, nonparallel annuli properly embedded in the complement of the lamination having the following property: One boundary component is the meridian of the knot and the other lies in some leaf of the lamination. Having been shown in Brittenham [4, Section 2], the existence of such a pair of annuli guarantees that non-trivial non-integral Dehn surgery on the knot cannot be exceptional. Also see $\mathrm{Wu}[34$, Section 3] about this arguments.

Lemma 2.5 On a hyperbolic arborescent knot of type II or III in $S^{3}$, all non-trivial exceptional surgeries are integral.

Proof In [33, Theorem 4.4], Wu showed that; all non-trivial non-integral surgeries on arborescent knots of type II give hyperbolic manifolds. Equivalently, an arborescent 
knot of type II has only integral non-trivial exceptional surgeries. Also, in [33, Theorem 3.6], Wu showed that; all non-trivial surgeries on arborescent knots of type III give hyperbolic manifolds. Equivalently, an arborescent knot of type III has no non-trivial exceptional surgeries.

Proof of Theorem 1.1 Let $K$ be a hyperbolic alternating knot in the 3-sphere. By Lemma 2.1, if $K$ has a connected prime alternating diagram $D$ satisfying $t(D)>4$, then all non-trivial non-integral surgeries on $K$ give hyperbolic manifolds. Otherwise, by Lemma 2.2, $K$ must be an arborescent knot. Following Wu [33], we divide arborescent knots into of type I, II, or III, and furthermore, divide type I knots into two-bridge knots or Montesinos knots of length three. Then we obtain that; all non-trivial exceptional surgeries are integral on a hyperbolic two-bridge knot by Lemma 2.3, on a Montesinos knot of length three by Lemma 2.4, and on an arborescent knot of type II or III by Lemma 2.5, respectively. This completes the proof.

\section{Remarks}

In this section, we collect some known facts about reducible/toroidal surgeries on alternating knots. As far as the author knows, there are no explicit studies on Seifert surgeries on alternating knots.

\subsection{Reducible surgeries}

We begin by considering reducible surgeries on alternating knots.

In fact, it is shown by Menasco and Thistlethwaite in [23, Corollary 1.1] that no Dehn surgeries on a hyperbolic alternating knot in $S^{3}$ yield reducible manifolds. We here include an outline of their arguments for completeness. They studied essential surfaces (ie, incompressible and boundary-incompressible) properly embedded in alternating knot exteriors, and established that the following holds for an essential surface $F$;

$$
-\chi(F) \geq \frac{1}{8} b \beta(n+2),
$$

where $b$ denotes the denominator of the boundary slope of $F, \beta$ the number of boundary components of $F$, and $n$ the twist-crossing number of the standard diagram of the knot. Thus if $F$ is of genus 0 , then $\beta-2 \geq \frac{1}{8} b \beta(n+2)$. From $\beta \geq 1$ and $b \geq 1$, this implies that $n \leq 5$. However, in [23], they determined alternating knots with twist-crossing number up to five ([23, Figure 1.2]), and they are in fact all two-bridge knots. For two-bridge knots, it is shown by Hatcher and Thurston in [15, Theorem 2(a)] 
that all non-trivial two-bridge knots other than $(2, p)$-torus knots have no reducible surgery.

In general, F Gonzàlez-Acuña and H Short conjectured in [12] that the only way to get a reducible $3-$ manifold by surgery on a knot in $S^{3}$ is to surger on a cable knot along the slope determined by the cabling annulus. This is now called the Cabling Conjecture, and still remaining open. See [18, Problem 1.79].

\subsection{Toroidal surgeries}

We next consider toroidal surgeries on alternating knots. Such surgeries are actually completely classified as follows.

In [24], based on the result obtained in [23], Patton claimed that if an alternating knot admits a toroidal surgery, then it is either a 2-bridge knot or a 3-strand pretzel knot. This is also achieved as [3, Lemmas 3.1 and 3.3]. We here include an outline of Patton's argument for completeness. Note that if a hyperbolic knot in $S^{3}$ yields a toroidal surgery, then the surgery slope actually becomes a boundary slope of an essential punctured torus. Then, from (1), we see that the denominator $b$ of the boundary slope of the punctured torus is at most 2 . However, if $b=2$, then the twist-crossing number must be at most 2 . Then we see that such knots are only $(2, p)$-torus knots, which are non-hyperbolic. If $b=1$, from (1), we see that the twist-crossing number of a hyperbolic alternating knot admitting toroidal surgery is at most 6. In [24, Section 1], Patton asserts that a hyperbolic alternating knot with twist-crossing number at most 6 must be a two-bridge knot or a Montesinos knot of length 3 . In the former case, in [24, Section 2], by using the machinery developed in [15], he showed that the 2-bridge knots have Conway forms of length two, ie, each of the knots is either of genus one, or bounds a once punctured Klein bottle. Also see Brittenham-Wu [5]. In the latter case, he studied in detail in [24, Section 3]. He used the machinery developed in Hatcher-Oertel [14], and showed that the Montesinos knots must be 3-strand pretzel knots. Again, each of such knots is either of genus one, or bounds a once punctured Klein bottle.

On general Montesinos knots, in [35] together with his previous results, Wu gave a complete classification of toroidal surgeries. Furthermore, he also gave a complete classification of toroidal surgeries on large arborescent knots, in [36].

Also remark that Gordon and Luecke proved in [13] that if a hyperbolic knot in $S^{3}$ admits a non-integral toroidal surgery, then the knot is one explicitly given by Eudave-Muñoz in [9]. 


\section{References}

[1] I Agol, Bounds on exceptional Dehn filling, Geom. Topol. 4 (2000) 431-449 MR1799796

[2] I Agol, Bounds on exceptional Dehn filling II arXiv:0803.3088

[3] S Boyer, X Zhang, Cyclic surgery and boundary slopes, from: "Geometric topology (Athens, GA, 1993)”, AMS/IP Stud. Adv. Math. 2, Amer. Math. Soc., Providence, RI (1997) 62-79 MR1470721

[4] M Brittenham, Exceptional Seifert-fibered spaces and Dehn surgery on 2-bridge knots, Topology 37 (1998) 665-672 MR1604911

[5] M Brittenham, Y-Q Wu, The classification of exceptional Dehn surgeries on 2-bridge knots, Comm. Anal. Geom. 9 (2001) 97-113 MR1807953

[6] C Delman, Essential laminations and Dehn surgery on 2-bridge knots, Topology Appl. 63 (1995) 201-221 MR1334307

[7] C Delman, Constructing essential laminations which survive all Dehn surgeries, preprint

[8] C Delman, R Roberts, Alternating knots satisfy Strong Property P, Comment. Math. Helv. 74 (1999) 376-397 MR1710698

[9] M Eudave-Muñoz, Non-hyperbolic manifolds obtained by Dehn surgery on hyperbolic knots, from: "Geometric topology (Athens, GA, 1993)", AMS/IP Stud. Adv. Math. 2, Amer. Math. Soc., Providence, RI (1997) 35-61 MR1470720

[10] D Gabai, Problems in foliations and laminations, from: "Geometric topology (Athens, GA, 1993)”, AMS/IP Stud. Adv. Math. 2, Amer. Math. Soc., Providence, RI (1997) 1-33 MR1470750

[11] D Gabai, U Oertel, Essential laminations in 3-manifolds, Ann. of Math. (2) 130 (1989) 41-73 MR1005607

[12] F González-Acuña, H Short, Knot surgery and primeness, Math. Proc. Cambridge Philos. Soc. 99 (1986) 89-102 MR809502

[13] C M Gordon, J Luecke, Non-integral toroidal Dehn surgeries, Comm. Anal. Geom. 12 (2004) 417-485 MR2074884

[14] A Hatcher, U Oertel, Boundary slopes for Montesinos knots, Topology 28 (1989) 453-480 MR1030987

[15] A Hatcher, W Thurston, Incompressible surfaces in 2-bridge knot complements, Invent. Math. 79 (1985) 225-246 MR778125

[16] K Ichihara, Integral non-hyperbolike surgeries, J. Knot Theory Ramifications 17 (2008) 257-261 MR2400664

[17] K Ichihara, I D Jong, S Mizushima, Seifert fibered surgeries on alternating Montesinos knots, in preparation 
[18] R Kirby (editor), Problems in low-dimensional topology, AMS/IP Stud. Adv. Math. 2, Amer. Math. Soc., Providence, RI (1997) MR1470751

[19] M Lackenby, Word hyperbolic Dehn surgery, Invent. Math. 140 (2000) 243-282 MR1756996

[20] M Lackenby, R Meyerhoff, The maximal number of exceptional Dehn surgeries arXiv:0808.1176

[21] W B R Lickorish, M B Thistlethwaite, Some links with nontrivial polynomials and their crossing-numbers, Comment. Math. Helv. 63 (1988) 527-539 MR966948

[22] W Menasco, Closed incompressible surfaces in alternating knot and link complements, Topology 23 (1984) 37-44 MR721450

[23] W Menasco, M B Thistlethwaite, Surfaces with boundary in alternating knot exteriors, J. Reine Angew. Math. 426 (1992) 47-65 MR1155746

[24] R M Patton, Incompressible punctured tori in the complements of alternating knots, Math. Ann. 301 (1995) 1-22 MR1312568

[25] G Perelman, The entropy formula for the Ricci flow and its geometric applications arXiv:math/0211159

[26] G Perelman, Ricci flow with surgery on three-manifolds arXiv:math/0303109

[27] G Perelman, Finite extinction time for the solutions to the Ricci flow on certain threemanifolds arXiv:math/0307245

[28] D Rolfsen, Knots and links, Mathematics Lecture Series 7, Publish or Perish, Berkeley, CA (1976) MR0515288

[29] P Scott, The geometries of 3-manifolds, Bull. London Math. Soc. 15 (1983) 401-487 MR705527

[30] M B Thistlethwaite, On the algebraic part of an alternating link, Pacific J. Math. 151 (1991) 317-333 MR1132393

[31] W P Thurston, The geometry and topology of three-manifolds (1980) Available at http://msri.org/publications/books/gt3m

[32] W P Thurston, Three-dimensional manifolds, Kleinian groups and hyperbolic geometry, Bull. Amer. Math. Soc. (N.S.) 6 (1982) 357-381 MR648524

[33] Y-Q Wu, Dehn surgery on arborescent knots, J. Differential Geom. 43 (1996) 171-197 MR1424423

[34] Y-Q Wu, Dehn surgery on arborescent knots and links-a survey, Chaos Solitons Fractals 9 (1998) 671-679 MR1628749

[35] Y-Q Wu, The classification of toroidal Dehn surgeries on Montesinos knots, to appear in Topology arXiv:math/0610870

[36] Y-Q Wu, Exceptional Dehn surgery on large arborescent knots arXiv: math/0610871 
School of Mathematics Education, Nara University of Education Takabatake-cho, Nara 630-8528, Japan ichihara@nara-edu.ac.jp http://mailsrv.nara-edu.ac.jp/ ichihara/index.html

Received: 17 August 2008 Revised: 5 October 2008 